
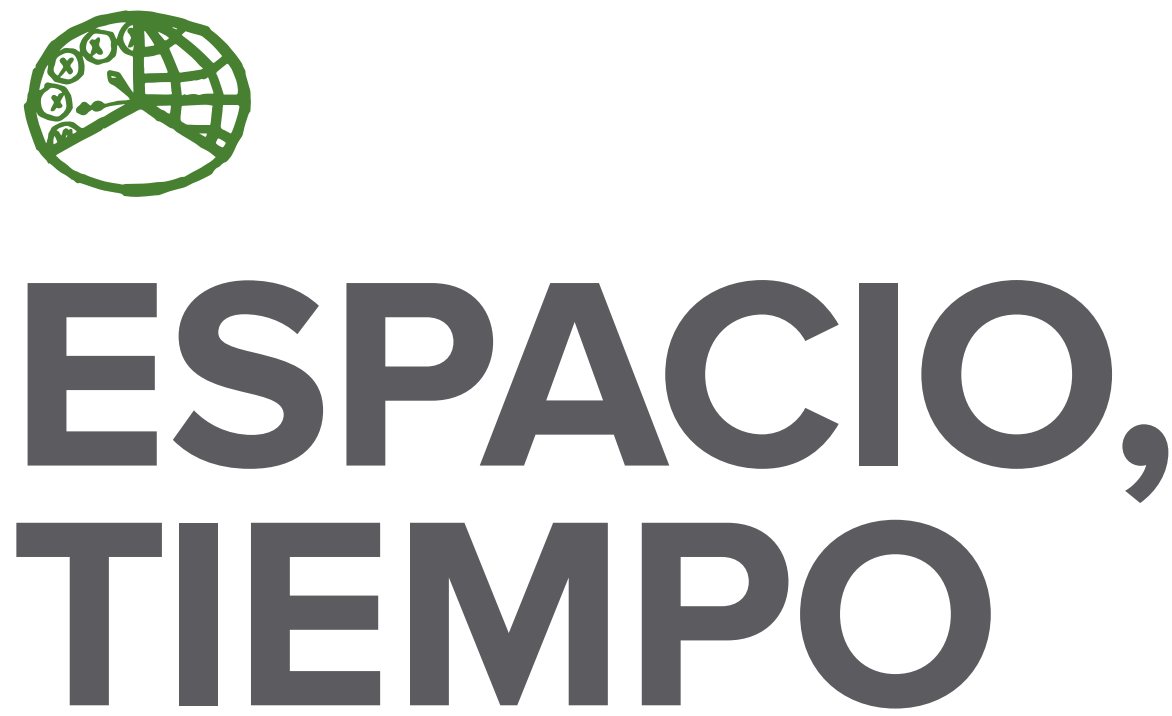

AÑOS 2013-2014

ISSN 1130-2968

E-ISSN 2340-146X
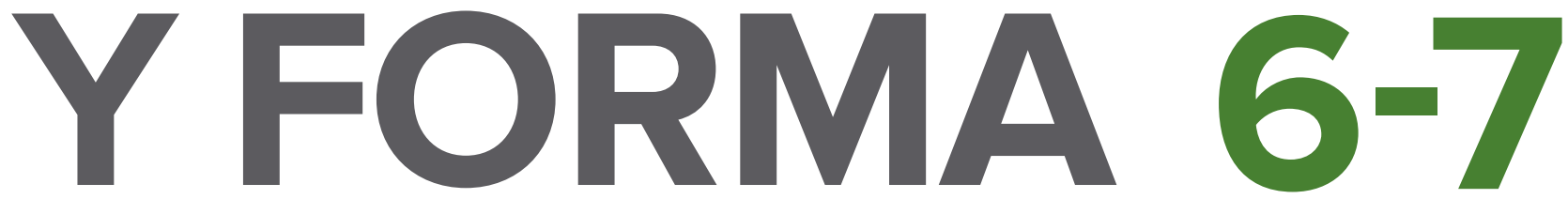

SERIE VI GEOGRAFÍA

REVISTA DE LA FACULTAD DE GEOGRAFÍA E HISTORIA 

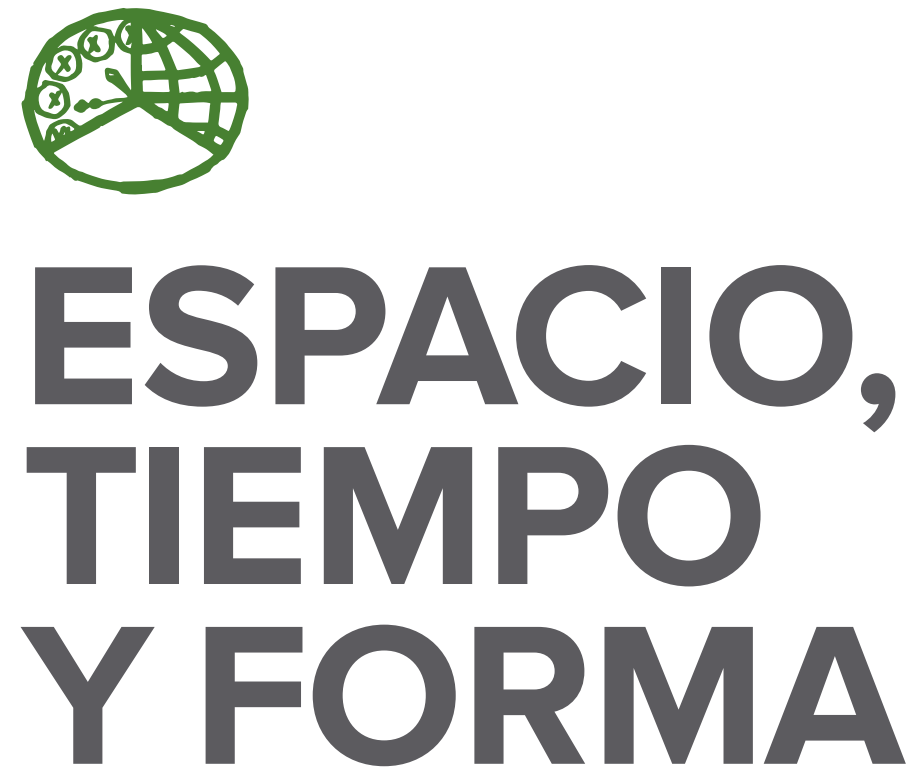

AÑOS 2013-2014

ISSN 1130-2968

E-ISSN 2340-146X

SERIE VI GEOGRAFÍA

REVISTA DE LA FACULTAD DE GEOGRAFÍA E HISTORIA

DOI: http://dx.doi.org/10.5944/etfvi.6-7.0

\section{UกED}

UNIVERSIDAD NACIONAL DE EDUCACIÓN A DISTANCIA 
La revista Espacio, Tiempo y Forma (siglas recomendadas: ETF), de la Facultad de Geografía e Historia de la UNED, que inició su publicación el año 1988, está organizada de la siguiente forma:

$$
\begin{aligned}
& \text { SERIE I - Prehistoria y Arqueología } \\
& \text { SERIE II - Historia Antigua } \\
& \text { SERIE III - Historia Medieval } \\
& \text { SERIE IV - Historia Moderna } \\
& \text { SERIE V - Historia Contemporánea } \\
& \text { SERIE VI - Geografía } \\
& \text { SERIE VII - Historia del Arte }
\end{aligned}
$$

Excepcionalmente, algunos volúmenes del año 1988 atienden a la siguiente numeración:

$$
\begin{aligned}
& \mathrm{N} .^{\circ} 1 \text { - Historia Contemporánea } \\
& \mathrm{N}^{\circ} 2 \text { - Historia del Arte } \\
& \text { N. }{ }^{\circ} 3 \text { - Geografía } \\
& \mathrm{N} .^{\circ} 4 \text { - Historia Moderna }
\end{aligned}
$$

ETF no se solidariza necesariamente con las opiniones expresadas por los autores.

Espacio, Tiempo y Forma, Serie vi está registrada e indexada, entre otros, por los siguientes Repertorios Bibliográficos y Bases de Datos: DICE, ISOC (CINDOC), RESH, IN-RECH, Dialnet, e-sPacio, UNED, CIRC, MIAR, FRANCIS, PIO, ULRICH'S, SUDOC, 2DB, ERIH (ESF).

\author{
UNIVERSIDAD NACIONAL DE EDUCACIÓN A DISTANCIA \\ Madrid, 2013-2014 \\ SERIE VI - GEOGRAFÍA N. ${ }^{\circ} 6-7,2013-2014$ \\ ISSN 1130-2968 · E-ISSN 2340-146x \\ DEPÓSITO LEGAL \\ M-21.037-1988 \\ URL \\ ETF VI · GEOGRAFÍA · http://revistas.uned.es/index.php/ETFVI \\ DISEÑO Y COMPOSICIÓN \\ Sandra Romano Martín · http://sandraromano.es \\ Ángela Gómez Perea · http://angelagomezperea.com \\ Impreso en España · Printed in Spain
}

(c) (i) (8) Esta obra está bajo una licencia Creative Commons

Reconocimiento-NoComercial 4.0 Internacional. 


\title{
REFLEXIONES AL FINALIZAR LA «DÉCADA DE LAS NACIONES UNIDAS DE EDUCACIÓN PARA EL DESARROLLO SOSTENIBLE 2005-2014»: REFERENCIAS AMBIENTALES EN LOS GRADOS DE LA UNED Y APORTACIÓN DE LA GEOGRAFÍA A LA EDS
}

\author{
REFLECTIONS AT THE END OF THE 'UNITED NATIONS \\ DECADE OF EDUCATION FOR SUSTAINABLE \\ DEVELOPMENT 2005-2014': ENVIRONMENTAL \\ REFERENCES IN THE DEGREE OF UNED \\ AND CONTRIBUTION OF GEOGRAPHY TO EDS
}

M. ${ }^{a}$ del Pilar Borderías Uribeondo ${ }^{1}$

Recepción: 1/12/2014 · Aceptación: 21/01/2015

DOI: http://dx.doi.org/10.5944/etfvi.6-7.0.14846

\begin{abstract}
Resumen
Las Naciones Unidas propusieron que en la década 2005-20I4 se promoviera un cambio en la educación para conseguir un futuro sostenible, cuyos resultados han sido valorados positivamente en la declaración de la Conferencia Mundial de la UNESCO sobre Educación para el Desarrollo Sostenible, celebrada en Aichi-Nagoya en noviembre de 20I4. En el ámbito universitario español, en el año 2005, la Conferencia de Rectores de las Universidades Españolas (CRUE) se sumó a este objetivo general de la década «integrar los valores inherentes al desarrollo sostenible en todos los aspectos del aprendizaje para motivar cambios de comportamiento que permitan una sociedad más sostenible y justa para todos», mediante la aprobación de las directrices para la Sostenibilización Curricular. En el ámbito geográfico, la Comisión sobre Educación Geográfica Internacional también se sumaba a la proclamada por la ONU «Década de las Naciones Unidas de Educación para el Desarrollo Sostenible 2005-20I4» con la Declaración de Lucerna 2007 sobre «Educación Geográfica para el Desarrollo Sostenible», confirmando así su compromiso con este objetivo y ampliando otras declaraciones anteriores, realizadas para impulsar el conocimiento del entorno mediante la educación geográfica. Con estas premisas, al finalizar esta década que ha querido impulsar la educación ambiental en todos los niveles de la enseñanza, en este artículo se hace un breve seguimiento de
\end{abstract}

1. Profesora Titular del Departamento de Geografía en la UNED.pborderias@geo.uned.es 
las referencias a la sostenibilidad ambiental en los objetivos y en las competencias generales y específicas que se expresan en los estudios de Grado en la UNED, con el fin de observar la repercusión que ha tenido esta iniciativa hasta el momento en esta universidad, y se reflexiona sobre las relaciones entre geografía, educación geográfica y desarrollo sostenible.

Palabras clave

educación para el desarrollo sostenible; sostenibilidad; educación superior; UNED; didáctica de la Geografía

\section{Abstract}

The United Nations established the decade of 2005-20I4 to promote the education for a sustainable future. The importance of this matter, was also recognized by the Worldwide Conference of UNESCO, which took place on Aichi-Nagoya on November 20I4. In the Spanish University educational program several efforts have been done to promote the educational future. For example, The Conference of Rectors from the Spanish Universities (also known as CRUE in Spanish) approved some guidelines for the sustainable curriculum in 2005. Also, The Commission for the International Education in Geography compromised with the education for a sustainable future by The Declaration of Lucerna. In this article we aim to establish the impact of the education for a sustainable future in the programs designed for undergraduate, and graduate degrees in the UNED. We also aim to make an effort to consider the relationships between geography, education in geography and sustainable development.

\section{Keywords}

education for sustainable development; sustainability; higher education; UNED; didactics of Geography 


\section{ALGUNAS REFERENCIAS SOBRE LA EDUCACIÓN PARA EL DESARROLLO SOSTENIBLE (EDS) EN LOS ESTUDIOS DE GRADO DE LA UNED}

Los objetivos de la Década 2005-20I4 de Educación para el Desarrollo Sostenible subrayaban la necesidad de integrar la perspectiva del desarrollo sostenible en todos los niveles del sistema educativo, de forma que la educación sea un agente para el cambio hacia la sostenibilidad. Se trataba de una propuesta generalizada de carácter transversal que implicara a toda la enseñanza en los objetivos ambientales, fuera cual fuese su objeto de conocimiento, con el fin de sensibilizar a los futuros profesionales sobre la situación medioambiental, relacionando la mejora ambiental, el desarrollo social y la economía sostenible, para buscar una salida hacia un mayor equilibrio.

Diversos aspectos ambientales y de sostenibilidad en la actual docencia universitaria en España están relacionados con esos objetivos, que se recogieron en las Directrices de la CRUE y en el Real Decreto 1393/2007, de 29 de octubre, por el que se establecía la ordenación de las enseñanzas universitarias oficiales. En ellos se expresa la necesidad de que la formación en cualquier actividad profesional contribuya al conocimiento y desarrollo de diferentes derechos y principios, entre los que se encuentra el de protección medioambiental.

En relación con estas pautas, uno de nuestros objetivos ha sido analizar, cuantificar y valorar la presencia de referencias ambientales y de sostenibilidad en los diferentes estudios de Grado que se imparten en la UNED, según la oferta académica del curso 2014-2015. Para ello se han revisado los estudios de Grado impartidos y se ha inventariado la información formal que se explícita en los programas oficiales, atendiendo a las alusiones a la sostenibilidad en los objetivos y en las competencias generales y específicas, así como la presencia de contenidos ambientales en las asignaturas, o el tratamiento de la relación Sociedad-Naturaleza.

De acuerdo con esta información, la presencia de referencias medioambientales en los objetivos, competencias y asignaturas de contenido ambiental en los diferentes Grados, se ha clasificado en tres niveles(presencia baja $<5 \%$; presencia media, entre 5-10\%; y presencia alta >10\%), en función del porcentaje de créditos con respecto al total de créditos del Grado correspondiente. Esta valoración nos ha permitido establecer comparaciones con los resultados de otras universidades en las que ya se ha realizado un estudio similar (Universidades de Valencia, Girona y Autónoma de Barcelona, estas dos últimas participantes en la Red ACES), habiéndose obtenido similares resultados en los estudios de Grado coincidentes. En la valoración aplicada a los Grados de la UNED, las referencias ambientales tienen una presencia alta en los Grados de Ciencias Ambientales y de Geografía e Historia; y una presencia media en los grados de Ciencias Químicas y Turismo; en el resto de los estudios de Grado su presencia queda por debajo del 5\%, aunque quedan muy próximos a este valor los Grados de la Facultad de Educación. En general, el análisis evidencia que una parte importante de los programas formativos presentan referencias, directas o indirectas, a las cuestiones medioambientales y de sostenibilidad (el 75\% de los Grados de la UNED muestran explícitamente su 
compromiso con la sostenibilidad), lo que supone un indicador de su importancia y de su gran transversalidad.

Los resultados obtenidos mostraron diferencias significativas en la sensibilización ambiental entre los distintos Grados, dado que en unos casos se muestra ampliamente competencias para la sostenibilidad pero en algunos no se hace ninguna referencia, ni siquiera a lo establecido en el RD I393/2007 en el que se especifica que se debe tener en cuenta que la formación en cualquier actividad profesional debe contribuir al conocimiento y desarrollo de diversos principios entre los que se encuentra el de protección medioambiental.

El contenido curricular de los diferentes estudios analizados, muestra que el $63 \%$ de los grados cuenta con alguna asignatura de contenido medioambiental. No obstante, es destacable que su presencia es especialmente relevante en los estudios que tratan directamente temas territoriales, como los Grados de Turismo y Geografía e Historia, en el Grado en Ciencias Químicas, en los Grados de Educación Social y Pedagogía, y, evidentemente, en el Grado de cc. Ambientales que, por su especificidad en los objetivos y contenidos del medio ambiente, es el de mayor relevancia en estas cuestiones.

A la vista de lo expuesto, se puede deducir que la introducción de criterios y competencias para la sostenibilidad avanza, aunque de forma dispar, y que si bien esta directriz fue asumida por la dirección de la universidad, su reflejo en las competencias del título correspondiente, puede depender de las Comisiones de los planes de estudio de cada titulación, según el grado de compromiso con la sostenibilidad de los miembros de cada una de ellas.

\section{LA DECLARACIÓN DE LUCERNA Y LA EDUCACIÓN PARA EL DESARROLLO SOSTENIBLE EN GEOGRAFİA}

La Declaración de Lucerna de 2007, proclamada por la Comisión sobre Educación Geográfica de la Unión Geográfica Internacional, constituyó una adhesión a la «Década de las Naciones Unidas de Educación para el Desarrollo Sostenible 2005-20I4", al considerarla una oportunidad para confirmar su compromiso con la educación para el desarrollo sostenible, y con ella se ampliaba, además, la Carta Internacional sobre Educación Geográfica (1992).

La reorientación de la educación geográfica hacia la EDS viene dada no sólo por los temas a enseñar y aprender (reseñados en la Agenda 2I.I), que aporten conocimientos relacionados con el desarrollo sostenible y sus tres vertientes: el medio ambiente, la sociedad y la economía; sino también por un conjunto de métodos para aprender, y contribuir al desarrollo de competencias, habilidades, actitudes y valores que inculquen el respeto hacia la naturaleza, los seres vivos, los derechos humanos, la justicia económica y la cultura de la paz.

Así, debe ofrecer a los alumnos la posibilidad de desarrollar actividades que promuevan una capacidad interpretativa y de valoración del entorno local; puede aportarles una conciencia espacial vinculada con el espacio geográfico; debe fomentar habilidades tales como saber comunicar, hacer predicciones, pensar de forma 
crítica; o adquirir una visión de los problemas en perspectiva, teniendo en cuenta su carácter cambiante y los diferentes puntos de vista; ejercitar su habilidad selectiva y discriminatoria para poder ordenar la gran cantidad de información existente; puede hacerles protagonistas de un comportamiento sostenible, y puede contribuir a la formación de ciudadanos críticos con los problemas ambientales.

La Declaración de Lucerna establece criterios básicos que orientan en la definición de objetivos de aprendizaje, y en la selección de temas, áreas geográficas objeto de estudio y modelos de enseñanza y aprendizaje, para la sostenibilización curricular de la Geografía. Estos son considerados esenciales para la educación sobre desarrollo sostenible en un currículo de Geografía (en las TABLAS i a 5 se exponen estos criterios ${ }^{2}$.

TABLA 1. COMPETENCIAS GEOGRÁFICAS PARA MEJORAR EL DESARROLLO SOSTENIBLE

RECOMENDADAS EN LA DECLARACIÓN DE LUCERNA

\section{COMPETENCIAS GEOGRÁFICAS ESPECÍFICAS}

* Conocimiento y comprensión geográfica

$\rightarrow$ Los sistemas naturales principales de la Tierra a fin de entender la interacción dentro y entre ecosistemas.

$\rightarrow$ Sistemas socioeconómicos de la Tierra para lograr un sentido de lugar.

$\rightarrow$ Conceptos espaciales: ideas-clave únicas de la Geografía que ayudan a los estudiantes a comprender el mundo: localización, distribución, distancia, movimiento, región, escala, asociación espacial, interacción espacial y cambio a lo largo del tiempo.

* Habilidades geográficas

$\rightarrow$ El uso de habilidades de comunicación, razonamiento y aptitudes prácticas y sociales para explorar temas geográficos en un rango de niveles desde lo local a lo internacional.

* Actitudes y valores

$\rightarrow$ Dedicación para buscar soluciones a preguntas y problemas locales, regionales, nacionales e internacionales sobre la base de la «Declaración Universal de Derechos Humanos».

TABLA 2. COMPETENCIAS INTERDISCIPLINARIAS PARA MEJORAR EL DESARROLLO SOSTENIBLE RECOMENDADAS EN LA DECLARACIÓN DE LUCERNA

Además de las competencias geográficas específicas, las habilidades interdisciplinarias cruciales para el desarrollo sostenible, a ser desarrolladas en colaboración con otras materias, son:

2. Fuente de las cinco tablas: Haubrich, H., Reinfried, S. \& SChleicher, Y. (2007) Declaración de Lucerna sobre Educación Geográfica para el Desarrollo Sostenible. 
* Reconocer problemas, evaluar alternativas, calcular riesgos.

* Percibir relaciones causa-efecto complejas y dinámicas.

* Reflexionar acerca de los efectos secundarios y las consecuencias que pueden esperarse de una acción.

* Pensar en sistemas y redes complejas.

* Encontrar, evaluar, procesar y usar información con métodos apropiados.

* Respetar otras visiones y opiniones.

* Pensar acerca de los motivos personales de cada uno y evaluarlos.

* Dar sentido y una base ética a la propia vida.

* Contribuir a tareas comunes con las propias competencias.

* Comprometerse con la planificación y los proyectos ambientales.

* Evaluar las acciones propias y sus resultados.

* Percibir el aprendizaje durante toda la vida como un enriquecimiento de la propia calidad de vida.

* Percibir problemas y fenómenos desde diferentes perspectivas.

* Aplicar flexiblemente diferentes métodos para resolver problemas.

* Relacionar experiencias locales y regionales con fenómenos globales.

TABLA 3. CRITERIOS PARA LA SELECCIÓN DE TEMAS GEOGRÁFICOS, SEGÚN LA DECLARACIÓN DE LUCERNA

* temas importantes en el mundo contemporáneo. Estos incluyen una selección de cuestiones concernientes al ser humano y a la naturaleza que son importantes para la vida, para un comportamiento espacial adecuado y para un comportamiento sostenible. Temas como el calentamiento global, el agotamiento de la energía, el excesivo uso de recursos no renovables, el cambio de la población y las disparidades globales pueden ser desarrollados. Es apropiada la consideración de conflictos resultantes de metas contradictorias concernientes a la sostenibilidad ambiental, económica y social.

* percepción geográfica del espacio, lugar y ambiente. Los temas incluyen provisión, uso, evaluación, formación y significación del espacio, lugar y ambiente.

* modos geográficos de observar la organización geográfica. Este criterio implica aproximaciones relacionadas con enfoques funcionales, sistémicos, prospectivos, de acción, estructura o proceso.

* ejemplos ilustrativos. Implican la selección de contenidos que sirvan como modelo referente a estructura/proceso relacionado con un tópico, que proporcionen ideas importantes y transferibles dentro de un problema; los contenidos son convenientes a la transferencia de ideas. 
* experiencias, inTeReses y pReconceptos estudiantiles. Implican la consideración de las experiencias, intereses y preconceptos estudiantiles de diferentes edades.

* significación para el individuo, la gente, la cultura y el ambiente. Este criterio incluye la importancia de problemas en contextos privados, públicos, políticos, profesionales o económicos.

* BALANCE. Involucra la selección de tópicos diferentes, contrastantes y multidimensionales y la consideración de perspectivas diversas de actores con intereses diferentes.

TABLA 4. CRITERIOS PARA LA SELECCIÓN DE ÁREAS GEOGRÁFICAS, SEGÚN LA

DECLARACIÓN DE LUCERNA

* ejemplos ilustrativos. Implican la selección de áreas significativas útiles para el aprendizaje de estructuras/procesos como modelos o que sean útiles para adquirir ideas transferibles.

* experiencias e intereses de los estudiantes. Este criterio tiene en cuenta el conocimiento de los estudiantes, sus intereses y experiencias en los distintos grupos de edades.

* significación. Implica considerar la posición política, económica o dimensional de un área y la consideración de su importancia ecológica/ambiental.

* VARIEDAd en extensión espacial. Implica tener presente la escala local, regional, nacional, internacional y global.

* BALANCE. Los temas incluyen la selección de áreas que son diferentes y contrastantes en términos de sus posiciones, tipos y medidas.

* cobertura topográfica. Este criterio apunta a temas que ayudan a aferrar la idea de que el espacio puede ser visto en forma general o temática - como una amplia grilla de orientación y una red de objetos topográficos individuales.

TABLA 5. CRITERIOS PARA LA SELECCIÓN DE ENFOQUES DE APRENDIZAJE, SEGÚN LA DECLARACIÓN DE LUCERNA

* Referencia a los intereses de los diferentes grupos de edad. Deben ser tomados en cuenta las preferencias e intereses de los diferentes grupos de edad.

* grado de las exigencias de aprendizaje. Significa que las exigencias sobre los estudiantes deberían incrementarse en volumen y en dificultad. El alumno debería realizar sus tareas con una independencia cada vez mayor.

* Series de aprendizaje de hechos conectados. Significa que los hechos relacionados o conectados deben ser ordenados de modo que se construyan unos sobre otros. 
* complejidad. Los contenidos y métodos empiezan desde el estudio de casos simples hasta la adquisición de los de mayor complejidad.

* abSTRacción. Se comienza a partir de fenómenos espaciales concretos y se avanza hacia modelos más abstractos.

* modos de observación. Este criterio implica que al comienzo del proceso de aprendizaje debería darse prioridad al modo de observación fisionómico, luego a la relación de procesos y finalmente a la forma funcional y prospectiva; métodos o enfoques constructivistas deberían ser utilizados para la comprensión de conceptos, procesos, teorías y espacio como constructos sociales cambiantes.

* inclusión de estudios de casos en contextos interRelacionados y VISIONES SINTÉTICAS. LOS ejemplos ilustrativos deben tener conexión con los contextos regionales.

* Secuencia regional. Significa que los tópicos regionales no deben ser estrictamente dispuestos desde lo cercano hacia lo lejano, sino en el sentido de una visión del mundo.

* extensión espacial. Significa tener en cuenta todos los niveles de escala, es decir la pequeña escala, la escala media así como también la dimensión internacional y global.

Como vemos, además de las competencias propias de la materia, que implica unas habilidades cognoscitivas (capacidad de comprender, y organizar ideas y pensamientos) es preciso desarrollar unas competencias transversales, unas competencias metodológicas (organizar el tiempo, estrategias para el aprendizaje, toma de decisiones, resolución de problemas y destrezas tecnológicas como gestión de la información y destrezas informáticas) y unas competencias personales, tanto individuales (capacidad de expresar los propios sentimientos; o habilidades críticas), como sociales (capacidad de trabajar en equipo, o capacidad de expresar un compromiso social o ético).

Con todo ello, la sostenibilidad se ofrece como una filosofía para guiar a las sociedades hacia un uso más racional de la Biosfera, lo cual implica indudables cambios estructurales no sólo de tipo económico, social, político, tecnológico, ético-cultural y científico, sino también educacional. Su introducción en las aulas supone un complemento a la filosofía didáctica clásica, de mejorar los conocimientos de las personas, aportando unas referencias éticas en las que el respeto por la conservación de la naturaleza y el medio ambiente juegue un papel importante. Su fuerza radica en que hoy día el concepto de desarrollo sostenible está relacionado con multitud de disciplinas que ponen de manifiesto problemas ambientales a los que intentan buscar soluciones.

La declaración de Lucerna ponía de manifiesto que la sostenibilidad entre naturaleza, economía y sociedad, es una clave conceptual básica para una educación geográfica, y en ella se destacaba que la adquisición de competencias geográficas puede mejorar la comprensión del desarrollo sostenible, poniendo de manifiesto la contribución de la Geografía a la educación para este objetivo. En ella se establecían 
unos criterios para desarrollar una Currícula Geográfica de Educación para el Desarrollo Sostenible, y se resaltaba la Importancia de las Tecnologías de la Información y la Comunicación (TiC).

La aportación de la Geografía al desarrollo sostenible abarca desde sus propios conocimientos conceptuales y espaciales, pasando por su metodología, su capacidad de interrelación y de síntesis, hasta conseguir que se desarrollen las competencias necesarias para actuar de forma sostenible en el territorio, y contribuir en la solución de problemas para la sostenibilidad. Aún más, podemos afirmar que existe una estrecha interrelación entre la Geografía y la sostenibilidad, pues ambos quedan profundamente relacionados con el ecosistema hombre-tierra.

Los conceptos geográficos básicos, como localización, distribución, distancia, movimiento, región, escala, asociación espacial, interacción espacial y cambio a lo largo del tiempo, proporcionan el conocimiento y la comprensión geográfica de los sistemas naturales, de la interacción entre ecosistemas, y de los sistemas socioeconómicos. Su modelo conceptual-metodológico, permite que se desarrollen habilidades cognitivas de observación, análisis y actuación en el entorno, al tiempo que se adquiere una capacidad para apreciar efectos de la acción humana sobre el medio ambiente, y se favorece el desarrollo de competencias a través de sus contenidos, que se contextualizan en un espacio y en una escala.

Los temas de estudio de la Geografía, abordan las adaptaciones, transformaciones e impactos que el hombre, como conjunto social, realiza sobre su entorno, e incorpora aspectos medioambientales. Podemos decir que la Geografía ha sido una de las ciencias sociales que más se ha interesado en estudiar los impactos, configuraciones y desequilibrios espaciales producidos por la escasa o inexistente aplicación de los principios del desarrollo sostenible en diversas áreas del planeta. Los temas de análisis geográfico con enfoque medioambiental son diversos, acercándose en muchos casos a la crítica con el entorno social y ambiental.

Los diversos enfoques geográficos, que intentan dar respuesta a aquellos problemas socioeconómicos, territoriales y ambientales que se han ido planteando, tienen una gran afinidad con la educación para la sostenibilidad (enfoques ecogeográfico, locacional, comportamental, crítico, sistémico), y actualmente todos ellos se interrelacionan y encuentran un plano de convergencia para explicar los diversos fenómenos socio-culturales que se producen en un espacio geográfico determinado. Desde un análisis crítico la Geografía, enseña a explicar la diversidad de espacios existentes, las desigualdades territoriales y sociales que se dan en el planeta y dentro de los propios países o naciones, y los efectos que la acción humana está teniendo sobre la naturaleza y el medio ambiente. Las nuevas formas de comprender y percibir el espacio, con una mirada crítica, o con los sentimientos y afectos que genera, o con la aceptación de la pluralidad de pensamiento y puntos de vista, ayudan a crear una ética geográfica.

La referencia espacial que ofrece la Geografía, aglutina en el territorio todas las dimensiones que concurren en la sostenibilidad (ecológica, económica, social, política, ética). Las situaciones positivas o negativas de sostenibilidad se producen sobre un territorio, y los distintos territorios ofrecen diversas características, que son producto de una serie de rasgos físico-ambientales y de las interacciones 
entre distintos grupos sociales y su entorno; y en función de esas características, se aplican las políticas y las estrategias de desarrollo, y, por tanto, sobre ese territorio se producirán las situaciones de sostenibilidad o insostenibilidad. Al mismo tiempo, la filosofía del desarrollo sostenible afecta a la planificación y a la ordenación territorial que se derive de los malos usos del territorio. Todo ello hace que el espacio geográfico constituya un marco de referencia indispensable en el análisis de la sostenibilidad, que aporte una base adecuada para diferenciar los objetivos de la sostenibilidad sobre los distintos territorios, y que sea condicionante de las aplicaciones para conseguirla.

Desde el punto de vista didáctico, esta perspectiva espacial permite la formación de la conciencia territorial, la explicación de los problemas medioambientales a todas las escalas (local, regional, nacional, continental y mundial) y facilita el desarrollo de competencias espaciales adecuadas para lograr un manejo sostenible de los ecosistemas. La escala global es hoy día muy importante, dado el complejo contexto de crisis económica, política y social, que interacciona a escala global, pero, como se expresaba en el Programa 2I de la UNESCO, en un mundo global es necesaria la combinación de distintas escalas geográficas porque al combinar la gran escala con la pequeña escala se obtiene una visión más compleja de la realidad y se puede establecer una explicación recíproca. Su aprendizaje exige un alto nivel de abstracción y es decisivo en la comprensión de los fenómenos de sostenibilidad. Esta dimensión territorial puede jugar un importante papel en el conocimiento y resolución de problemas ambientales, estudiando el impacto de la acción humana en el aumento de los desastres, en la preservación de la naturaleza, en la conservación de las condiciones que demandan las nuevas generaciones, o en la organización del espacio geográfico, permitiendo que los estudiantes ejerciten y desarrollen habilidades tendentes a la búsqueda de soluciones a estos problemas, desde distintos enfoques y perspectivas.

Las nuevas tecnologías constituyen una importante herramienta en Geografía, permitiendo la enseñanza de sistemas de información geográfica y la realización de mapas que pueden reflejar espacialmente los problemas ambientales.

En definitiva, en el aprendizaje de la Geografía es posible no sólo analizar las interrelaciones e interconexiones de los distintos objetos que conforman un sistema espacial y facilitar la comprensión del entorno, sino también formar ciudadanos con una conciencia medioambiental, que se sume a la concienciación y solidaridad de las nuevas generaciones. Trabajar desde una óptica geográfica facilita una visión amplia y una capacidad de síntesis que resulta adecuada para el estudio del desarrollo sostenible, lo que, sin duda, contribuye a que los estudiantes reciban formación en competencias para la sostenibilidad, que les ayudará a enfrentarse a los problemas ambientales en su vida profesional.

\section{CONCLUSIONES}

Desde que en la Cumbre de la Tierra de Río de Janeiro en I992 se plantease la necesidad de reorientar los sistemas educativos hacia la sostenibilidad, hasta finales 
del año 20I4, se ha avanzado sin duda en estos objetivos, pues es indiscutible que se ha producido una aceptación internacional para impulsar y generalizar la preocupación por la sostenibilidad de nuestro planeta desde las aulas, en todas las materias y desde todos los niveles de la enseñanza. Pero tal vez se han quedado cortas las previsiones y queden aún muchos ámbitos donde no se atienda al enfoque de la sostenibilidad, como vienen indicando algunos estudios.

Queda todavía un largo camino para alcanzar una formación sostenible integral, como manifestaron los participantes en la Conferencia Mundial de la UNESCO sobre la EDS, celebrada en Aichi-Nagoya el 3 de noviembre de 20I4, los cuales, tras confirmar el progreso realizado, pidieron que se tomaran medidas urgentes para fortalecer y ampliar en mayor medida la EDs. En este sentido, una estrategia para el año 2015 es la Agenda de Desarrollo Post-2015, que comprenderá, entre otros compromisos, el inicio de la implementación del Programa Global de Acción Mundial (GAP) de Educación para el Desarrollo Sostenible de la UnEsCo, el cual presenta dos objetivos relacionados con la enseñanza y el aprendizaje: fortalecerlos en todos los programas y actividades que promuevan el desarrollo sostenible, y reorientarlos para que todos puedan adquirir conocimientos, competencias, valores y aptitudes que les permitan contribuir al desarrollo sostenible.

Desde la universidad se está impulsando la conciencia sobre la fragilidad del medio ambiente y el uso incontrolado de los recursos, mediante su función generadora de conocimiento e impulsora de modelos científicos, sociales y humanistas. La universidad puede ejercer de agente dinamizador del cambio para la sostenibilidad, ya que forma a futuros profesionales, los cuales, en el desarrollo de su profesión, podrán ejercer un efecto directo e indirecto sobre su entorno. Son muchas las universidades que ya han iniciado, en mayor o menor medida, este proceso, aunque el hecho de que la EDS se plantee como una manera de vivir y entender la vida ofrece algunos debates, puesto que no todos los docentes conciben la sostenibilidad de esta manera, sino sólo como un concepto y contenido importante.

Desde la perspectiva geográfica, la educación para la sostenibilidad cuenta con muchas posibilidades por su implicación en el territorio, sobre el que se produce todo tipo de interrelaciones. Su aportación a la tarea multidisciplinar de la sostenibilidad y del desarrollo sostenible no sólo constituye un enfoque más a la amplia gama de interpretaciones y visiones que suscita el desarrollo sostenible; sino que constituye una herramienta eficaz para el entendimiento y comprensión de los procesos de sostenibilidad, contribuyendo, a la conformación y al diseño de actitudes y modelos dirigidos hacia este objetivo.

Las reflexiones y argumentaciones sobre la incidencia de la Educación para el Desarrollo Sostenible en la universidad, y las oportunidades que ofrece para ello la Geografía, vertidas en este artículo, pretenden ser una revisión de la utilidad de la Geografía como Ciencia para la Sostenibilidad, ya sea a niveles teóricos, conceptuales, metodológicos, o aplicados, y aportar un reconocimiento al interés que tiene el desarrollo sostenible en el quehacer geográfico. 


\section{BIBLIOGRAFÍA}

Araya Palacios, F.R., «Didáctica de la geografía para la sustentabilidad (2005-2014)». Revista de Teoría y Didáctica de las Ciencias Sociales II (2006), pp. 27-6I.

Aznar, P. \& UlL, M. A A, «La formación de competencias básicas para el desarrollo sostenible: el papel de la universidad». Revista de Educación, $n .^{\circ}$ extraordinario «Educar para el desarrollo sostenible»(2009), pp. 219-238.

Aznar Minguet, P., «El reto educativo de la sostenibilidad en el marco del espacio europeo de la educación superior», en Escolano BENITO, A.: Educación superior y desarrollo sostenible. Madrid, Biblioteca Nueva, 2006.

Aznar Minguet, P. \& Ull, M.A. «Universidad y Sostenibilidad: Formación en competencias básicas». Revista Educación. Ministerio de Educación, 2009.

Bodini, H, «Los nuevos desafíos de la geografía». Geoespacios i8. p. 49. La Serena-Chile: Ediciones Universidad de la Serena. 200I.

de la Calle Carracedo, Mercedes, La enseñanza de la Geografía ante los nuevos desafíos ambientales, sociales y territoriales. Universidad de Valladolid. Disponible en internet http://ifc.dpz.es/recursos/publicaciones/33/36/03delacalle.pdf

CARIDE, J.A, La educación para el desarrollo sostenible: realidades y perspectivas desde el Decenio Naciones Unidas-UNESCO (2005-20I4). En L.E. 2006.

CRUE Directrices para la Sostenibilización Curricular CRUE. Documento aprobado por el Comité Ejecutivo del Grupo de Trabajo de Calidad Ambiental y Desarrollo Sostenible de la CRUE, celebrado en Valladolid el I8 de abril de 2005. http://www.uah.es/universidad/ Ecocampus/documentos/C3SostenibilizacionCurricular_CRUE.pdf.

Gelı, A.M. ${ }^{\mathrm{a}}$, «Universidad, sostenibilidad y ambientalización curricular». En Ambientalización Curricular en los Estudios Superiores. Arbat, E. \& Geli, A.M. eds. Tomo I. Aspectos Ambientales de las Universidades. Universitat de Girona. Servei de Publicacions / Red ACES, 2002.

Geli, A.M. ${ }^{\text {, }}$ Junyent, M. \& SÁnchez, S. (eds.), Ambientalización curricular de los estudios superiores. Tomo IV. Acciones de intervención y balance final del Proyecto Universidad de Girona. Publicaciones de la RED-ACES) 2004.

Durán D., Daguerre, C. \& LARA, A. Los cambios mundiales y la enseñanza de la geografía. Editorial Troquel Educación. Buenos Aires, Argentina. 1996.

Durán, Diana. Las dimensiones de la sustentabilidad. Fundación Educa Ambiente.20Io. [Documento en línea] Disponible: http://www.ecoportal.net/content/view/full/91572.

GonzÁlez, J. \& WagenaAR, R. «Tuning Educational Structures in Europe» Informe final. Proyecto piloto. 2003

GranAdos SÁNCHEZ, J. «La recerca en l'ensenyament de la geografia per al desenvolupament sostenible». Documents d'anàlisi geográfica. vol. 56/2, 2010, pp. 339-356.

- «La educación para la sostenibilidad en la enseñanza de la geografía. Un estudio de caso». Enseñanza de las Ciencias Sociales. Revista de Investigación, Io, 20II, pp. 3I-43.

Gurevich, R., Blanco, J., Fernández, M.V. \& Tobío, O. «Notas sobre la enseñanza de una geografía renovada». I998. Ed. AIQUE. 
Haubrich, H., Reinfried, S. \& Schleicher, Y. «Declaración de Lucerna sobre Educación Geográfica para el Desarrollo Sostenible». Publicada a través de la página de la Comisión. Lucerna. 2007.

MARRón GAITE, M.J. «Desarrollo sostenible, globalización y educación en valores ambientales desde la geografía. Una propuesta metodológica en el marco europeo de Educación Superior». Valencia: Grupo de Didáctica de la AGE, Universidad de Valencia, 2007, pp. I33-I46.

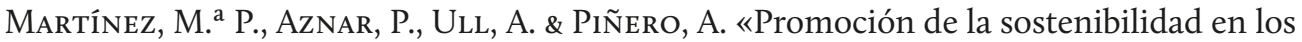
currícula de la enseñanza superior desde el punto de vista del profesorado: un modelo de formación por competencias». Educatio Siglo XXI (Murcia), 25, 2007, pp. 187-208.

Martínez-Agut, M.P., Ull, M.A., Piñero, A., Palacios, B. \& Aznar Minguet, P. «Análisis de las referencias a la sostenibilidad en los nuevos títulos universitarios» VI Jornadas de investigación en docencia universitaria. Universidad de Alicante. 2008.

Melendro, M., Murga, M. ${ }^{a}$ A., Novo, M. \& Bautista-Cerro, M. ${ }^{a}$ J. (2008) «Estrategias formativas innovadoras en Educación Ambiental y para el Desarrollo Sostenible», Revista Iberoamericana de Educación a Distancia (RIED) II, pp. 15-40.

NoguÉ, J. \& Romero, J. «Otras geografías, otros tiempos. Nuevas y viejas preguntas, viejas y nuevas respuestas», en NoguÉ, J. \& Romero, J. (eds.), Las otras geografías. Valencia: Tirant lo Blanch, 2006, pp. 15-53.

Souto, X.M. «Fines y objetivos en la enseñanza de la Geografía: los condicionantes sociales y epistemológicos». En Prats, J. (coord.), Didáctica de la Geografía y la Historia. Barcelona, Graó, 20II, pp. II5-I29.

TORo SÁnchez, F.J., «El desarrollo sostenible: un concepto de interés para la Geografía». Cuadernos Geografícos. Universidad de Granada. Vol. 40. 2007, pp. I49-I8I.

Unesco. Decenio de las Naciones Unidas de la Educación para el Desarrollo Sostenible 200520I4: Plan de aplicación internacional. Proyecto. París: UNESCO. 2005.

UnEsCo (2009). Educación para el Desarrollo Sostenible. Objetivos. febrero de 2009 Disponible en: $h$ ttp://portal.unesco.org/education/es/ev.php.

Unión Geográfica Internacional, Comisión de Educación Geográfica. Declaración Internacional sobre Educación Geográfica. 1992. Disponible en: http://www.raco.cat/ index. php/RevistaGeografia/article/viewFile/46086/56892.

- Comisión de Educación Geográfica. Declaración Internacional sobre Educación Geográfica para la diversidad cultural. 2000. Disponible en: $h t t p: / / w w w . a g e-g e o g r a f i a . e s / v 2 / d i v e r s i-$ dad_cultural.php?TB_iframe=true\&hei ght $=430$ \&width $=750$.

- Comisión de Educación Geográfica. Declaración Internacional sobre Educación Geográfica para el desarrollo sostenible. 2007. Disponible en: $h t t p: / / w w w . i g u-c g e . o r g / C h a r t e r s-p d f /$ spanish.pdf. 


\section{Artículos · Articles}

19 María del Pilar Borderías URIBEondo

Reflexiones al finalizar la «Década de las Naciones Unidas de Educación para el Desarrollo Sostenible 2005-2014»: referencias ambientales en los Grados de la UNED y aportación de la Geografía a la EDS / Reflections at the end of the 'United Nations Decade of Education for Sustainable Development 2005-2014': environmental references in the Degree of UNED and contribution of Geography to ESD

\section{Jesús Calzadilla, José Luis López \& Aurelio VilLa}

Análisis espacial del desarrollo económico rural a nivel territorial / Spatial analysis at territorial level of rural economic development

47 David Cocero Matesanz, José Miguel Santos Preciado, María del Carmen Muguruza Cañas, fernando Santa Cecilia Mateos, maría Victoria Azcárate luxán, María del Pilar Borderías Uribeondo \& María Eugenia Prieto Flores

La utilización en los estudios urbanos de la cartografía catastral y su manejo mediante un SIG: aplicación al municipio de Getafe (Madrid) / The use in urban studies of cadastral mapping and management through GIS: application to the town of Getafe (Madrid)

73 Juan antonio Fernández-Cañadas López-Peláez, María Palomo Segovia \& LUis Pantoja Trigueros

Delimitación espacial de las zonas probables de salida de aludes en el macizo de Peñalara mediante el uso de SIG / Spatial delimitation of the possible output zones of avalanches in the Peñalara massif using GIS techniques

Jesús Miguel García Rodríguez

Importancia patrimonial y territorial del legado hidroeléctrico en el curso bajo del Río Saja (Cantabria) / Patrimonial and territorial importance of hydroelectric legacy in the lower reaches of Saja (Cantabria)

\section{Beatriz Martínez de Teresa}

Paisaje urbano y problemática medioambiental: el caso del distrito de Retiro (Madrid) / Cityscape and environmental issues: the case of Retiro district (Madrid)

antonio Martínez Puche, Salvador Martínez Puche, joaquín Palací SOler \& Vicente Zapata Hernández

Estrategias territoriales participativas como instrumentos de diagnóstico y prospectiva, en el contexto europeo de los fondos de cohesión 2014-2020: el caso de Villena (Alicante) / Territorial participatory strategies as instruments of diagnosis and prospective, in the European context of the funds of cohesion 2014-2020 in Villena (Alicante)
185 Francisco José Morales Yago

Evolución de la jerarquía urbana en la Región de Murcia tras más de treinta años de la aprobación del estatuto de autonomía (1982-2014) / Evolution of urban hierarchy in the Region of Murcia thirty years after the adoption of the statute of autonomy (1982-2014)

205 José Luis Rodríguez García

Conservación y gestión del paisaje en el Campo de Albacete / Landscape conservation and management in the Campo de Albacete

227 XANTI SUdUPE Zabalo

El abandono en el paisaje de periferia y periurbano de Bilbao / Abandonment in the periphery and peri-urban landscape of Bilbao

257 M. Antonio Zárate Martín \& InÉs Ortíz Álvarez

Estrategias para la conservación y gestión sostenible de los paisajes culturales urbanos en México y España / Strategies for the conservation and sustainable management of the urban cultural landscapes in México and Spain

\section{MARÍA DEL CARMEN ZORRILLA LASSUS}

La puesta en valor del paisaje a través de la educación, propuesta para el desarrollo de la Isla de Vieques (Puerto Rico) / The enhancement of landscape through education proposal for the development of the island of Vieques (Puerto Rico)

\section{Recensión de un libro · Book Review}

315 Borderías Uribeondo, M.P. \& Muguruza Cañas, M.C.: Impacto ambiental 6101301GRoiAol. (María José Aguilera ArilLa)

\section{Imágenes y palabras · Pictures and words}

323 Francisco josé Morales Yago

Portmán (La Unión-Cartagena): ¿Crónica de un impacto medioambiental en vías de solución? / Portmán (La Unión-Cartagena): chronicle of an environmental impact way of solution?

\section{Comentario de material didáctico · Comment of didactic material}

Mendoza Vargas, Héctor (coord.): Estudios de geografía humana de México. (Alejandro García Ferrero)

Historia de la Geografía Española • History of Geography in Spain 335 Nota preliminar

337 Aurelio Nieto Codina

Paisaje y Geografía en la obra de Eduardo Hernández-Pacheco / Landscape and Geography in the work of Eduardo Hernández-Pacheco 